\title{
Increased leptin by hypoxic-preconditioning promotes autophagy of mesenchymal stem cells and protects them from apoptosis
}

\author{
WANG LiHan, HU XinYang, ZHU Wei, JIANG Zhi, ZHOU Yu, CHEN PanPan \& \\ WANG JianAn* \\ Cardiovascular Key Lab of Zhejiang Province; the Second Affiliated Hospital, School of Medicine, Zhejiang University, Hangzhou 310009, \\ China
}

Received July 29, 2013; accepted October 11, 2013; published online January 21, 2014

\begin{abstract}
Autophagy is the basic catabolic progress involved in cell degradation of unnecessary or dysfunctional cellular components. It has been proven that autophagy could be utilized for cell survival under stresses. Hypoxic-preconditioning (HPC) could reduce apoptosis induced by ischemia and hypoxia/serum deprivation (H/SD) in bone marrow-derived mesenchymal stem cells (BMSCs). Previous studies have shown that both leptin signaling and autophagy activation were involved in the protection against apoptosis induced by various stress, including ischemia-reperfusion. However, it has never been fully understood how leptin was involved in the protective effects conferred by autophagy. In the present study, we demonstrated that HPC can induce autophagy in BMSCs by increased LC3-II/LC3-I ratio and autophagosome formation. Interestingly, similar effects were also observed when BMSCs were pretreated with rapamycin. The beneficial effects offered by HPC were absent when BMSCs were incubated with autophagy inhibitor, 3-methyladenine (3-MA). In addition, down-regulated leptin expression by leptin-shRNA also attenuated HPC-induced autophagy in BMSCs, which in turn was associated with increased apoptosis after exposed to sustained H/SD. Furthermore, increased AMP-activated protein kinase phosphorylation and decreased mammalian target of rapamycin phosphorylation that were observed in HPC-treated BMSCs can also be attenuated by down-regulation of leptin expression. Our data suggests that leptin has impact on HPC-induced autophagy in BMSCs which confers protection against apoptosis under H/SD, possibly through modulating both AMPK and mTOR pathway.
\end{abstract}

BMSCs, autophagy, hypoxic-preconditioning, leptin, apoptosis

Citation: Wang LH, Hu XY, Zhu W, Jiang Z, Zhou Y, Chen PP, Wang JA. Increased leptin by hypoxic-preconditioning promotes autophagy of mesenchymal stem cells and protects them from apoptosis. Sci China Life Sci, 2014, 57: 171-180, doi: 10.1007/s11427-014-4607-4

Although severe hypoxia can lead to cell death for certain cell types such as endothelial cells, repeated episodes of short periods' exposure to hypoxia (hypoxic-preconditioning) have shown conferring cytoprotective benefits. Research done in our laboratory and others has revealed that hypoxic-preconditioning protects bone marrow-derived mesenchymal stem cells (MSCs) from apoptosis and enhances cell survival after implantation [1,2]. However, the mechanisms underlying the beneficial effects of hypoxia

*Corresponding author (email: jian_an_wang@yahoo.com) preconditioned MSCs remain unclear.

Autophagy is the cellular process responsible for the degradation and removal of damaged organelles and protein aggregates [3]. Autophagy is critical to cell survival. The interruption of autophagy may initiate severe energy metabolism disorder and cell death [4]. Many scientists have reported that activation of autophagy protects MSCs from apoptosis [5]. Recent cancer research demonstrates that hypoxia activates autophagy and promotes tumor cell survival [6]. We hypothesized that hypoxic-preconditioning induces autophagy of bone marrow-derived MSCs (BMSCs), and it 
activates autophagy to protect MSCs from hypoxia and serum deprivation.

Recently, research data from our lab and others suggested that hypoxia induces leptin expression [7,8]. Leptin, the $16-\mathrm{kD}$ nonglycosylated polypeptide product of the $o b$ gene, is synthesized mainly by white adipose [9]. The best-known function of leptin is to regulate appetite. Leptin acts on cells in hypothalamus to reduce the production of orexigenic neuropeptides and reciprocally enhance the secretion of anorectic peptides, thus controlling food intake [10]. Beyond its function as a satiety factor, leptin has a wide range of biological functions, including reproduction, immunity, angiogenesis, and anti-apoptotic effects. Recent reports of in vivo administration of leptin activating AMP-activated protein kinase (AMPK) phosphorylation in peripheral tissues suggests that it may influence autophagy [11,12]. In vitro studies demonstrated that leptin stimulated autophagy in cultured human or mouse cell lines, a phenomenon that was linked to the activation of AMPK as well as the inhibition of mammalian target of rapamycin (mTOR) [13].

The objective of this study was to find out whether leptin has any contribution to increased autophagy induced by hypoxic-preconditioning in BMSCs. Increased autophagy protects stem cells from apoptosis under sustained hypox$\mathrm{ia} /$ serum deprivation (H/SD).

\section{Materials and methods}

\subsection{Cell culture}

Bone marrow was harvested from the tibia and femur of C57/BL6 mouse (20-25 g, male) and seeded into cell culture flasks with DMEM (Gibco, USA) containing 10\% FBS (Gibco) and $1 \%$ penicillin-streptomycin (Gibco) at $37^{\circ} \mathrm{C}$ in a humidified atmosphere containing 5\% $\mathrm{CO}_{2}$. After $24 \mathrm{~h}$, non-adherent cells were discarded, adherent cells were washed three times with phosphate buffered saline (PBS). Fresh complete medium was added. When the cells reached $80 \%$ confluence, they were detached using $0.25 \%$ trypsin-EDTA (Gibco) and subcultured at the ratio of $1: 2$. All cells used in the experiment were passage 3 .

\subsection{Hypoxic preconditioning and H/SD}

For hypoxia treatment, cells were plated at $5 \times 10^{4}$ cells per well in 6-well plates in complete culture medium and incubated under hypoxia condition $\left(0.5 \% \mathrm{O}_{2}, 5 \% \mathrm{CO}_{2}\right)$ using ProOX Model C21 system (BioSpherix, USA) for 6, 12 and $24 \mathrm{~h}$, respectively. After hypoxic preconditioning, the MSCs were washed with PBS, and incubated under hypoxia condition $\left(0.5 \% \mathrm{O}_{2}, 5 \% \mathrm{CO}_{2}\right)$ with serum-free medium for $48 \mathrm{~h}$. 3-methyladenine (3-MA, at $5 \mathrm{mmol} \mathrm{L}^{-1}$ from SigmaAldrich, USA) and rapamycin $\left(25 \mathrm{mmol} \mathrm{L}^{-1}\right.$ from Cell Signal Technology, USA) were added to inhibit or induce au- tophagy, respectively, to further investigate the role that autophagy activation plays in protection apoptosis in MSCs.

\subsection{Infecting BMSCs with leptin targeting shRNA}

For the leptin-knockdown assay, low passage number mouse BMSCs were transduced with a lentiviral vector carrying mouse leptin shRNA (LV-LEP-RNAi, purchased from Shanghai SBO Medical Biotechnology Co. Ltd. (Shanghai, China)). $5 \times 10^{5}$ BMSCs were seeded and incubated at $37^{\circ} \mathrm{C}, 5 \% \mathrm{CO}_{2}$ overnight. At $\sim 70 \%$ confluency, $0.5 \mathrm{~mL}$ of filtered lentiviral supernatant $\left(5 \times 10^{7}\right.$ titer units $)$ containing $8 \mathrm{mg} \mathrm{mL}^{-1}$ Polybrene (Sigma, USA) was added (MOI=100). After $24 \mathrm{~h}$, the virus-containing medium was replaced with fresh medium. To select infected cells, puromycin $\left(2.5 \mu \mathrm{g} \mathrm{mL}^{-1}\right)$ was added and incubated for $24 \mathrm{~h}$. According to Leptin mRNA sequence (NM_008493), Leptin targets shRNA constructs. The target oligonucleotides were designed at the position in gene sequence from 897 gene site. Two cDNA template chains were designed and Age I, EcoR I enzyme cleavage sites were introduced at the terminals. The sequences of shRNA that directed against the mice Leptin were as follows: sense, 5'- CCGGTCTGCAGGACATTCTTCAATTCAAGAGATTGAAGAATGTCCT GCAGATTTTTTG-3'; antisense, 5'-AATTCAAAAAATCTGCAGGACATTCTTCAATCTCTTGAATTGAAG AATGTCCTGCAGA-3'. The sequences of scrambled shRNA were sense, 5'-CCGGTTCTCCGAACGTGTCACGTTTCAAGAGAACGTGACACGTTCGGAGAATTTT TG-3'; antisense, 5'-AATTCAAAAATTCTCCGAACGTGTCACGTTCTCTTGAAACGTGACACGTTCGGAGAA-3'. The double-stranded oligonucleotides were cloned into the linear vector containing enhanced green fluorescent protein (EGFP) gene.

\subsection{Immunofluorescence staining}

Cells were fixed with $4 \%$ paraformaldehyde for $10 \mathrm{~min}$ and washed with PBS. The cells were then incubated with blocking solution (DAKO, USA) for $30 \mathrm{~min}$ at room temperature to inhibit internal factors in cells followed by an overnight incubation with rabbit anti-mouse LC3 (1:200) (Cell Signal Technology) at $4^{\circ} \mathrm{C}$. The samples were then stained with secondary antibody (Dylight 488 abcam) for $1 \mathrm{~h}$ and Hoechst 33258 staining for $15 \mathrm{~min}$ at room temperature. Images were detected and analyzed using a Leica DM550B microscope (Leica Microsystems CMS GmbH, Germany).

Cells were detached from the plates and fixed with $2.5 \%$ glutaraldehyde in $0.1 \mathrm{~mol} \mathrm{~L}^{-1}$ phosphate buffer at $4^{\circ} \mathrm{C}$ for 2 h. After washed with PBS, cells were post-fixed with $1 \%$ osmic acid at $4{ }^{\circ} \mathrm{C}$ for $1.5 \mathrm{~h}$. Cells were then dehydrated by a serial gradient ethanol and then embedded in Embed-812 medium (Electron Microscopy Sciences, USA). Ultrathin sections were cut using an Ultrotome (Leica, Reichert Ul- 
tracuts, Germany) on uncoated copper grids and stained with $0.2 \%$ lead citrate/ $1 \%$ uranyl acetate. Images were recorded under a transmission electron microscope (JEM1230; JEOL, Japan)

\subsection{Western blotting}

Equal amounts of protein $(40 \mu \mathrm{g} / \mathrm{lane})$ were separated by electrophoresis for Western blotting analysis. The proteins were transferred for $2.5 \mathrm{~h}$ at $250 \mathrm{~mA}$ onto PVDF membranes (Bio-Rad, UK), and the membranes were blocked for $1 \mathrm{~h}$ at room temperature in 5\% skim milk. The membranes were then incubated overnight at $4{ }^{\circ} \mathrm{C}$ with primary antibodies. The following primary antibodies were used: phosphorylated (Thr172) and total AMPK, phosphorylated (serine 2448) and total mTOR, beclin1, light chain 3 (LC3) of microtuble-associated protein 1 , cleaved caspase- 3 , and $\beta$-actin from Cell Signal Technology. The membranes were washed and incubated for $1 \mathrm{~h}$ at room temperature in blocking solution containing peroxidase-conjugated secondary antibodies. Then the membranes were washed again and processed for analysis by using a Chemiluminescence Detection Kit (Millipore, USA) as instructed by the manufacturer.

\subsection{Terminal deoxynucleotidyl transferase-mediated dUTP nick end-labeling (TUNEL) assay}

TUNEL assay was performed using In Situ Cell Death Detection Kit, TMR red (Roche Applied Science, USA) according to manufacturer's procedure. Briefly, treated cells grown on 24-well plates with cover slips in each well were fixed in $4 \%$ paraformaldehyde, then permeabilized using $0.1 \%$ TritonX-100 in $0.2 \%$ sodium citrate and incubated in TUNEL reaction mixture for $1 \mathrm{~h}$ at $37^{\circ} \mathrm{C}$. Cover slips were mounted on glass slides with Vectashield mounting medium with DAPI (1.5 $\mu \mathrm{g} \mathrm{mL}^{-1}$, Vector Laboratories, Inc., USA) and analyzed by fluorescence microscopy to observe DNA fragmentation. Quantification of TUNEL positive cells was performed using Image Pro.

\subsection{Statistical analysis}

Data were analyzed using SPSS 15.0 software. All values were presented as mean \pm SD. Differences among groups were tested by one-way analysis of variance. Two-sided $P$-values were used and $P<0.05$ was considered statistically significant.

\section{Results}

\subsection{Autophagy was induced in BMSCs after hypoxic preconditioning}

To investigate hypoxic preconditioning-induced autophagy in BMSCs, we detected the levels of the type II LC3
(LC3-II), a biochemical marker of autophagy, by Western blotting. Under normal condition, LC3 protein exists in the cytosol as type I (LC3-I). When autophagy is activated, LC3-I can be recruited by autophagosome membrane and converted to LC3-II [14]. As shown in Figure 1A, compared with the control group (normoxic-treated), the ratio of LC3-II/LC3-I was significantly increased after HPC (6-12 h) and autophagy promoter rapamycin $\left(25 \mathrm{nmol} \mathrm{L}^{-1}\right)$ treatment for $6 \mathrm{~h}$, although the expression of autophagic marker beclin1 had no significant difference among groups. Moreover, treatment with autophagic inhibitor 3-MA $\left(5 \mathrm{mmol} \mathrm{L}^{-1}\right)$ attenuated the HPC-induced autophagy. We also performed an immunofluorescence assay to evaluate LC3, which is an early marker for autophagosome. As shown in Figure 1C, the green dots represent LC3 that was detected in the cytoplasm of BMSCs. Compared to the control group, the expression of LC3 increased after HPC (6-12 h) and being treated with rapamycin $\left(25 \mathrm{nmol} \mathrm{L}{ }^{-1}\right)$. This suggested that HPC elicited autophagosomes. However, HPC-elicited autophagosomes decreased after 3-MA treatment.

\subsection{HPC-induced autophagy protected BMSCs from apoptosis under H/SD condition}

To investigate the association of autophagy and apoptosis, we treated BMSCs with autophagy promoter rapamycin or hypoxic preconditioned with or without autophagy inhibitor 3-MA, and then exposed them to H/SD for $24 \mathrm{~h}$. As shown above, the autophagic activity in BMSCs was induced significantly in the groups that had HPC (6-12 h) and rapamycin treatment compared to the control group. This was showed by higher LC3-II/LC3-I ratio (Figure 1A) and more autophagosome formation (Figure 1C). 3-MA suppressed the autophagic activity induced by HPC in BMSCs. Additionally, apoptosis analysis performed via terminal deoxynucleotidyl TUNEL staining indicated that enhancing BMSCs autophagy by rapamycin and HPC significantly attenuated the apoptosis ratio of BMSCs under H/SD (Figure $2 \mathrm{~B}$ ) when compared to the control group. 3-MA-treated BMSCs showed a higher rate of apoptosis (Figure 2B). We also found the expression of cleaved caspase-3 was enhanced by $3-\mathrm{MA}$ while reduced by rapamycin and HPC significantly (Figure 2A). These results suggested that HPC-induced autophagy played a protective role in BMSCs apoptosis under H/SD.

\subsection{Leptin- knockdown attenuated HPC induced au- tophagy of BMSCs}

It has been reported that HPC increased the expression of leptin which could induce autophagy in several cell lines under certain conditions. To test whether leptin was able to modulate autophagy in HPC-BMSCs, we used shRNA to down-regulate leptin expression in BMSCs, then exposed BMSCs to HPC for $12 \mathrm{~h}$. As shown in Figure 3A, LC3-II, 

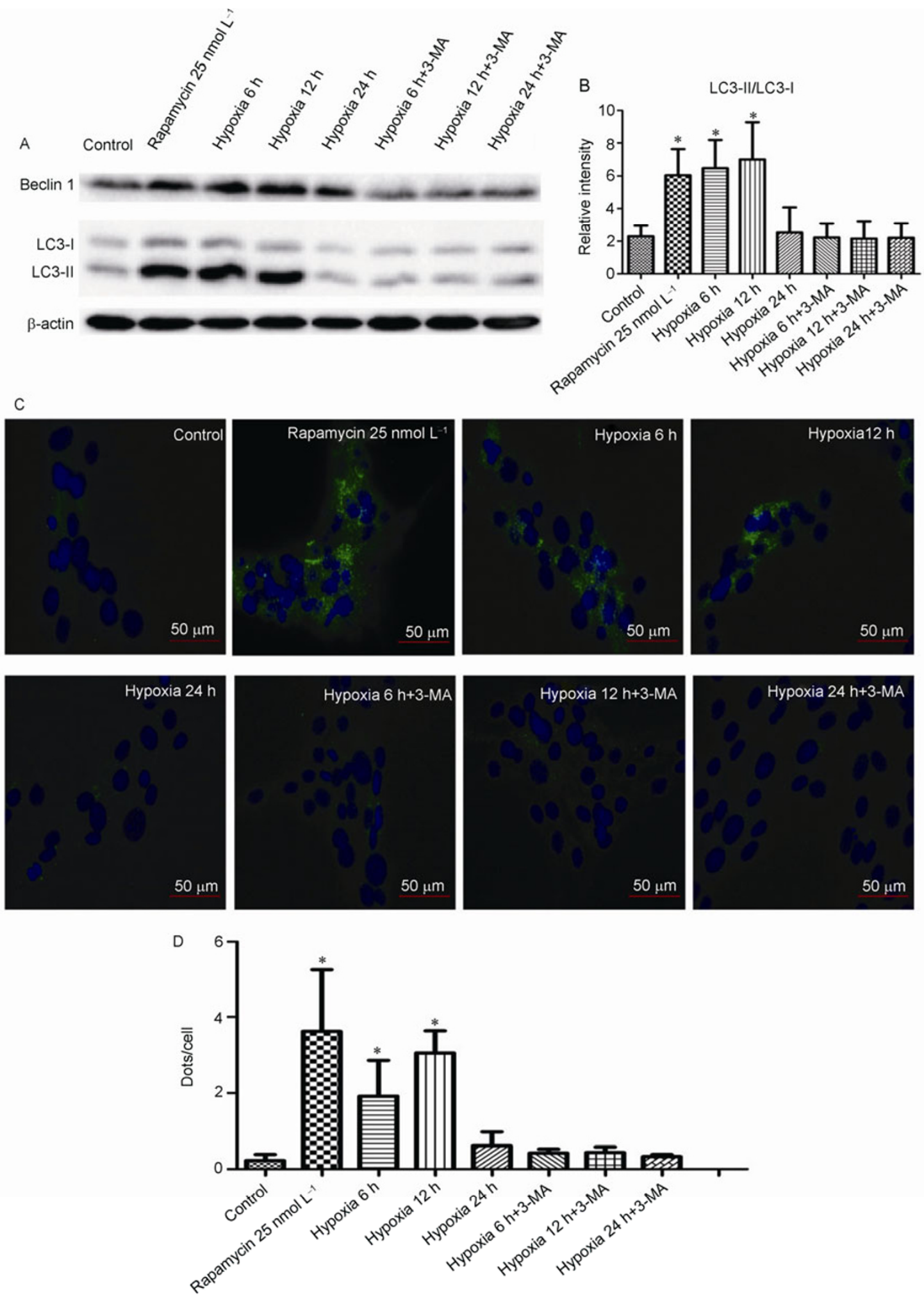

Figure 1 Hypoxic-preconditioning induces autophagy in BMSCs. A, Western blotting analysis of BMSCs showed that hypoxic-preconditioning (for 6 and $12 \mathrm{~h}$ ) induced the expression of autophagic marker LC3-II which was abrogated by 3-MA and induced by Rapamycin, compared to normoxic-treated control group. Beclin1 expression had no significant difference. B, Quantification of LC3-II/LC3-I was performed using the immunoreactive bands with Quantity One ${ }^{\circledR}$. C, Cellular LC3 which represents autophagysome detected by immunofluorescence in BMSCs showed that hypoxic-preconditioning (for 6 and 12 h) increased LC3 expression which was abrogated by 3-MA and induced by Rapamycin (the green dots represent LC3 and blue one represents nucleus). D, Quantification of the staining of LC3 particles (green dots) obtained from immunofluorescence was performed with Image Pro. Figures are representative images of at least three different samples, and each experiment was repeated at least thrice. Results are expressed as mean $\pm \mathrm{SD}$. *, $P<0.05$ vs. Control, Hypoxia 24 h, Hypoxia 6 h+3-MA, Hypoxia 12 h+3-MA and Hypoxia 24 h+3-MA. 

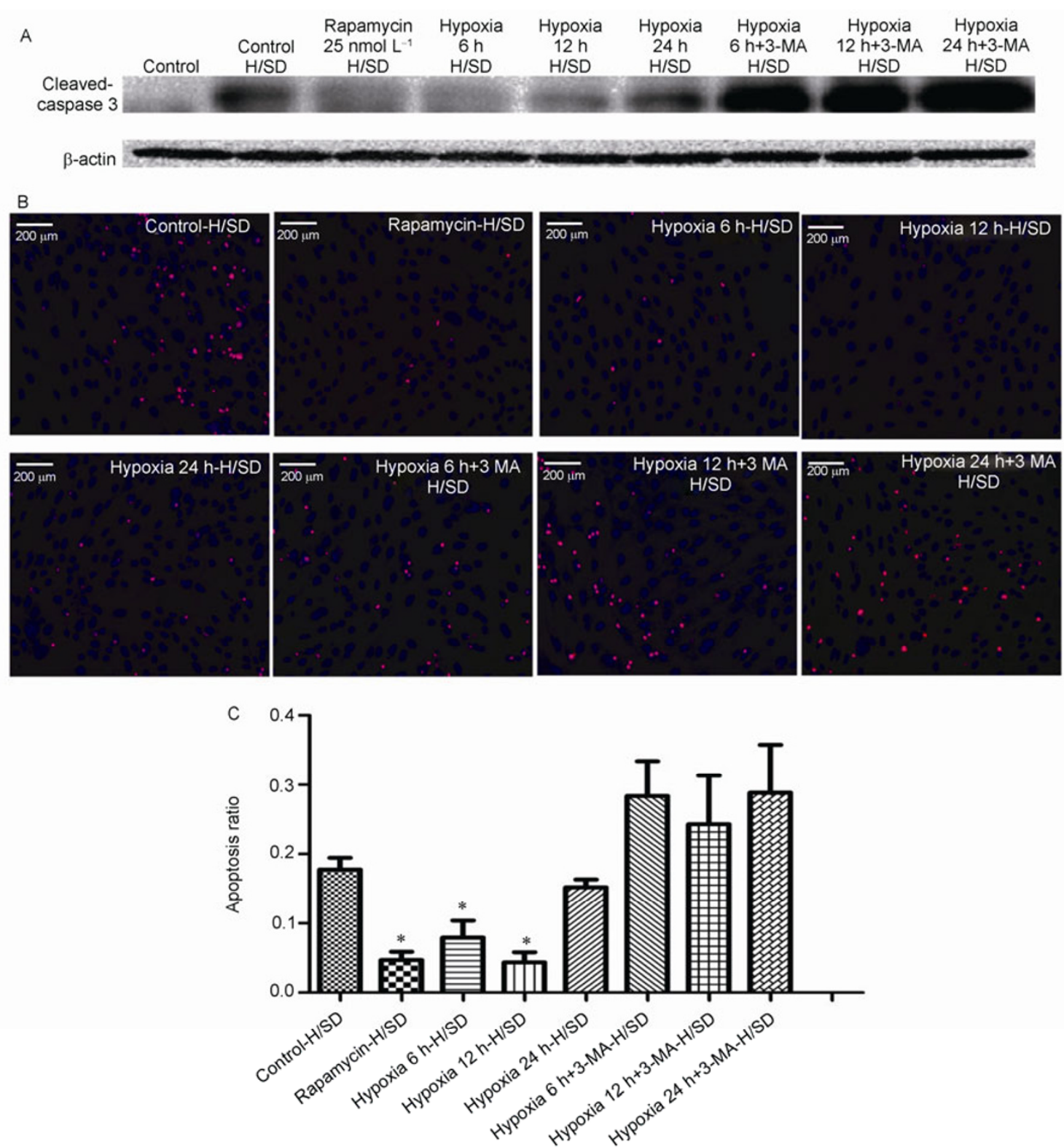

Figure 2 Enhanced autophagic activity attenuates apoptosis. A, Western blotting analysis of levels of cleaved form of caspase 3 in BMSCs demonstrated that hypoxic-preconditioning (for 6 and $12 \mathrm{~h}$ ) and rapamycin treatment attenuates H/SD induced apoptosis. Meanwhile, 3MA abrogated the protective effect. B, Terminal deoxynucleotidyl transferase dUTP nick end labelling (TUNEL) immunofluorescence analysis of BMSCs showed that hypoxic-preconditioning and rapamycin treatment attenuates the apoptosis ratio of BMSCs under H/SD while 3-MA abolished the protective effect. Fluorescent microscopic images show the TUNEL staining (red) and nuclear staining (blue). C, Quantification of apoptosis of BMSCs using the TUNEL stained images with Image Pro software. Figures are representative images of at least three different samples and each experiment was repeated at least thrice. Results are expressed as mean \pm SD. $*, P<0.05$ vs. Control, Hypoxia 24 h, Hypoxia 6 h+3-MA, Hypoxia 12 h+3-MA and Hypoxia 24 h+3-MA.

identified by Western blot, elevated in HPC-WT (wild type) and $\mathrm{NC}$ (negative control) groups when compared to the control group. However, leptin-knockdown led to a decrease of the LC3-II in HPC BMSCs, whereas this negative effect was absent when rapamycin was added (Figure 3A). In addition, we found that the number of autophagosomes observed in HPC-Leptin- knockdown-BMSCs was significantly decreased compared with HPC-WT and NC groups. This decrease could be reversed by rapamycin treatment (Figures 3C and 4). These results suggested that leptin is a key factor for the enhanced autophagic activity in MSCs exposed to HPC.

\subsection{Leptin knockdown attenuated the protective effect} of HPC-induced autophagy against apoptosis in H/SDtreated BMSCs

We have confirmed that leptin plays an important role in HPC-induced autophagy in BMSCs. To further test the function of leptin in the protective effects of autophagy, 

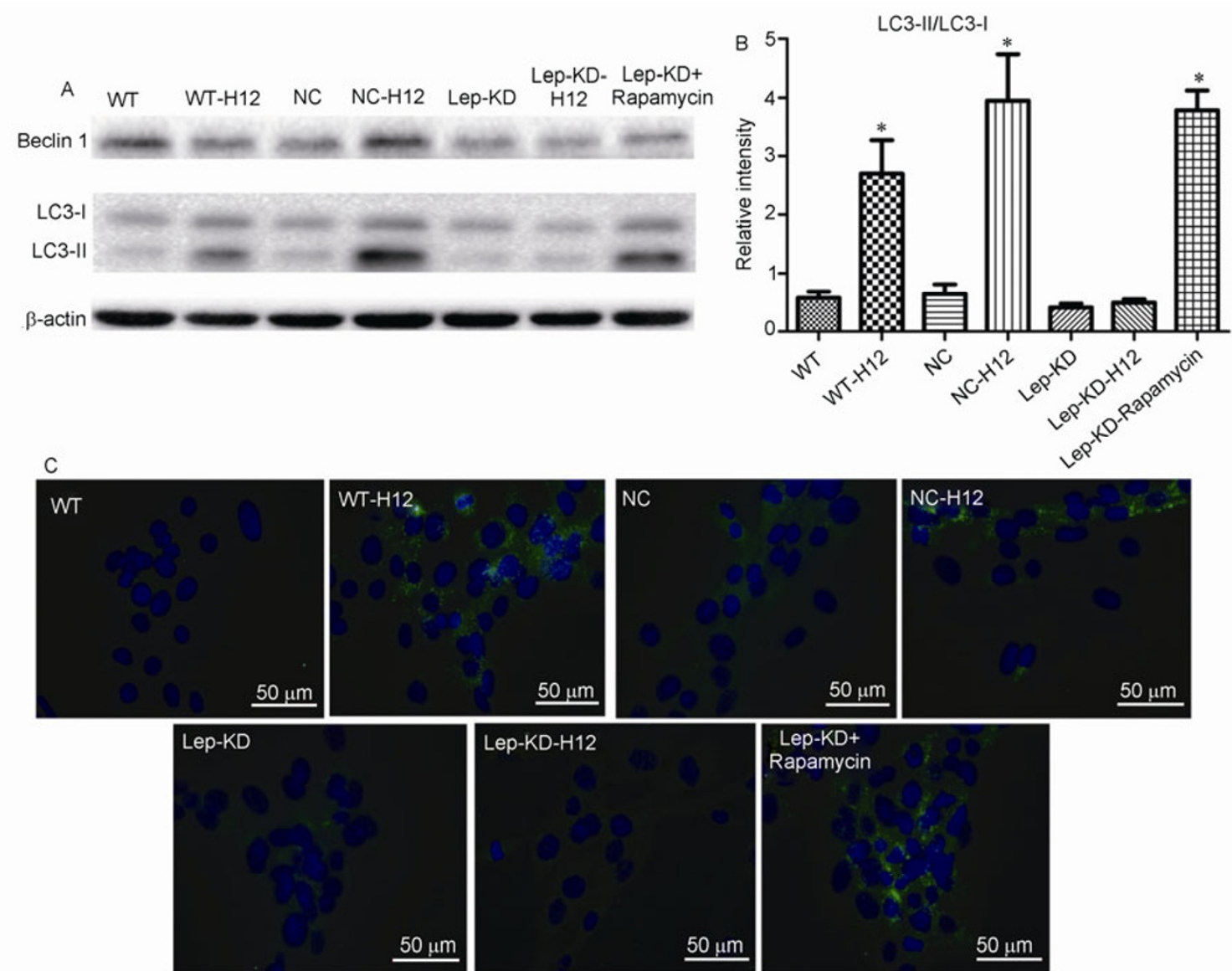

$\underline{50 \mu \mathrm{m}}$
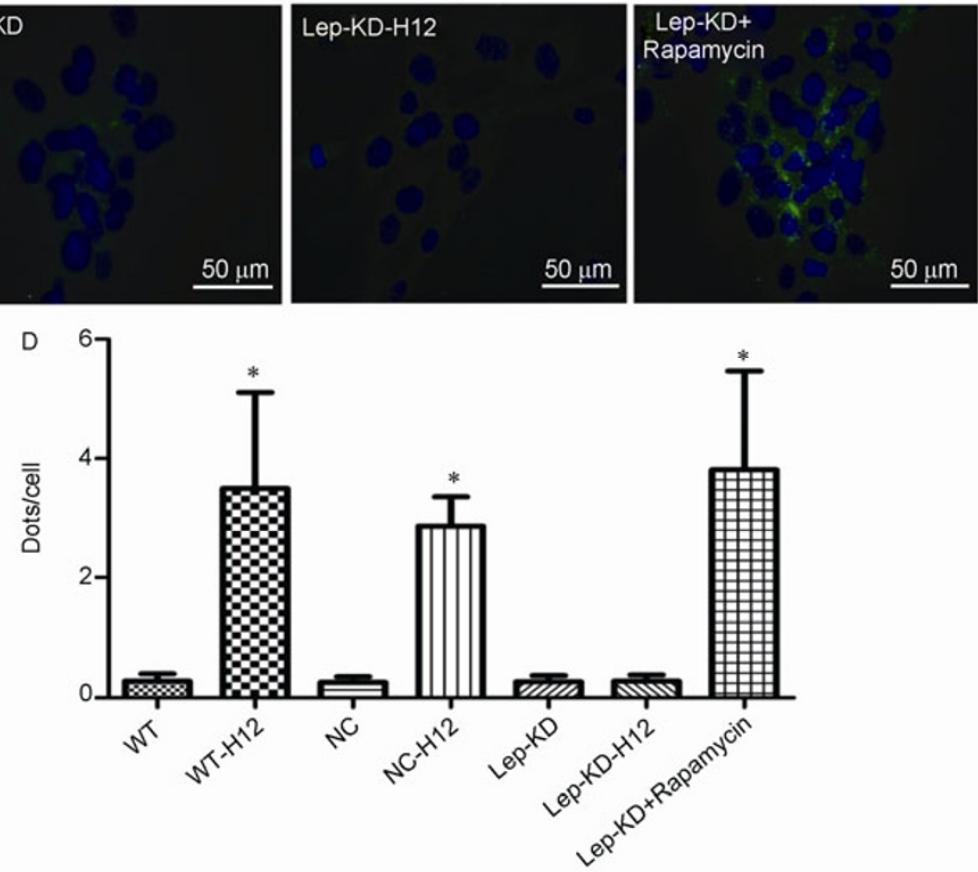

Figure 3 leptin-knockdown reduce autophagic activity induced by hypoxic-preconditioning in BMSCs. A, Western blotting analysis of autophagic marker LC3-II in wild type, negative control, leptin-knockdown BMSCs showed that leptin-knockdown abrogated hypoxic-preconditioning induced autophagy while rapamycin could reverse this phenomenon. B, Quantification of LC3-II/LC3-I was performed using the immunoreactive bands with Quantity One ${ }^{\circledR}$. C, Cellular LC3 detected by immunofluorescence in wild type, negative control, leptin-knockdown BMSCs demonstrated that leptin-knockdown reduced hypoxic-preconditioning induced LC3 which was reversed by rapamycin (the green dots represent LC3 and blue one represents nucleus). D, Quantification of the staining of LC3 particles (green dots) obtained from immunofluorescence was performed with Image Pro. Figures are representative images of at least three different samples, and each experiment was repeated at least thrice. Results are expressed as mean \pm SD. *, $P<0.05$ vs. WT (wild type), NC (negative control), Lep-KD (leptin-knockdown) and Lep-KD-H12 (leptin-knockdown BMSCs treated with hypoxic-preconditioning for 12 h).

leptin-knockdown BMSCs were hypoxic preconditioned for $12 \mathrm{~h}$ with or without rapamycin, then exposed to H/SD for 24 h. As shown in Figure 5B, the ratio of apoptosis identified by TUNEL assay demonstrated that HPC protected WT and NC BMSCs from apoptosis induced by H/SD. However, while leptin knockdown attenuated the anti-apoptotic ca- pacity of HPC, rapamycin treatment restored the protective effect (Figure 5B). From Western blot analysis, we found that leptin knockdown also led to the increase in cleaved caspase- 3 at $24 \mathrm{~h}$ of H/SD in HPC BMSCs compared to that of the groups that had HPC-WT, HPC-NC, and rapamycin treatment (Figure 5A). These results indicated that leptin 


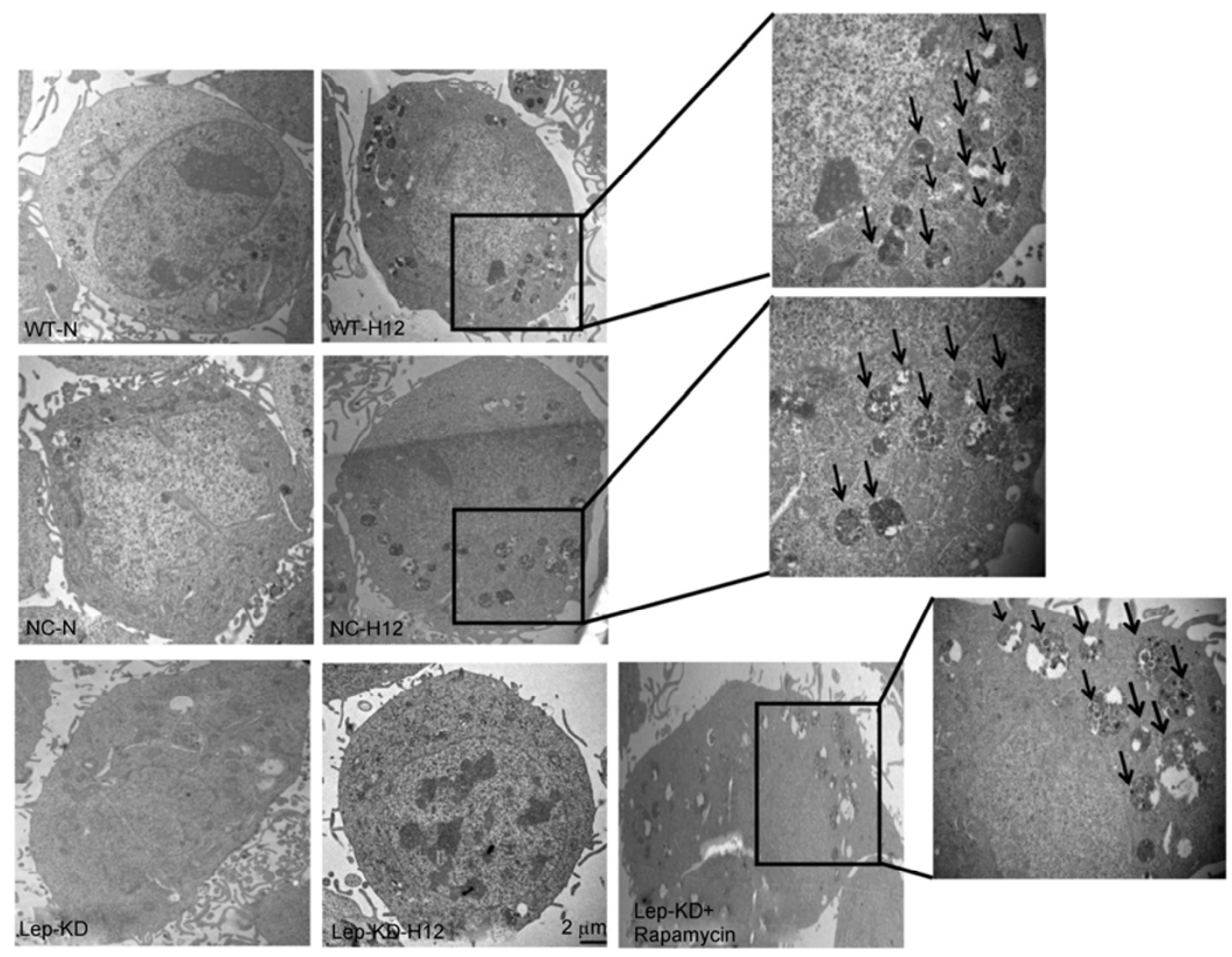

Figure 4 Transmission electron micrograph of BMSCs. Electron micrographs showed that leptin-knockdown decreased the double-membraned autophagic vacuoles (the arrows indicated) which was induced by hypoxic-preconditioning.

might play an important role in the protective effect of HPC-induced autophagy.

\subsection{HPC-induced autophagy regulated by leptin was associated with AMPK/mTOR pathway}

Several studies have shown that AMPK/mTOR pathway plays a role in regulating autophagy. During the experiment, we also investigated whether the AMPK/mTOR pathway is associated with HPC-induced autophagy, which is regulated by leptin. The results from Western blot indicated that HPC did stimulate the activation of phosphorylation of AMPK $\alpha$ as well as the dephosphorylation of mTOR after $12 \mathrm{~h}$. However, leptin knockdown abrogated the effects of HPC on both AMPK phosphorylation and mTOR dephosphorylation which could be reversed by autophagy promoter rapamycin (Figure 6). These results suggest that AMPK/ mTOR pathway might play a role in the stimulating function of leptin on HPC induced MSC autophagy.

\section{Discussion}

Scientists have been studying stem cells as useful tools for regenerative medicine. Stem cells have the potential to pro- tect, repair, and regenerate impaired tissues. Working with embryonic stem cells has many drawbacks, such as genetic mutations, teratomas formation, difficult ethical considerations, and possibility of immune rejection [15]. To overcome these limitations, MSCs have been studied as an alternative source for cell therapy, because they are easy to prepare, have immunologic advantages, and involve no ethical controversy [16]. However, cell transplantation application with MSCs is hampered by early cell death, partially due to oxygen or nutrient deprivation; therefore how to enhance MSCs survival has received much attention.

In our current study, the results showed that autophagy was promoted in BMSCs after hypoxic preconditioning (peaked at $12 \mathrm{~h}$ ) and it served as a protector for BMSCs apoptosis under H/SD. Leptin may regulate HPC-induced autophagy via AMPK/mTOR pathway. To our knowledge this is the first report showing that leptin regulates HPC-induced autophagy in BMSCs, which protects BMSCs from apoptosis under H/SD.

Autophagy plays key roles in cellular physiology, such as degradation of long-lived proteins, organelle turnover, adaptation to nutrient depletion, and cellular homeostasis consistency $[17,18]$. Autophagy which has contributions to cell survival or death in response to a stressor can be dramati- 


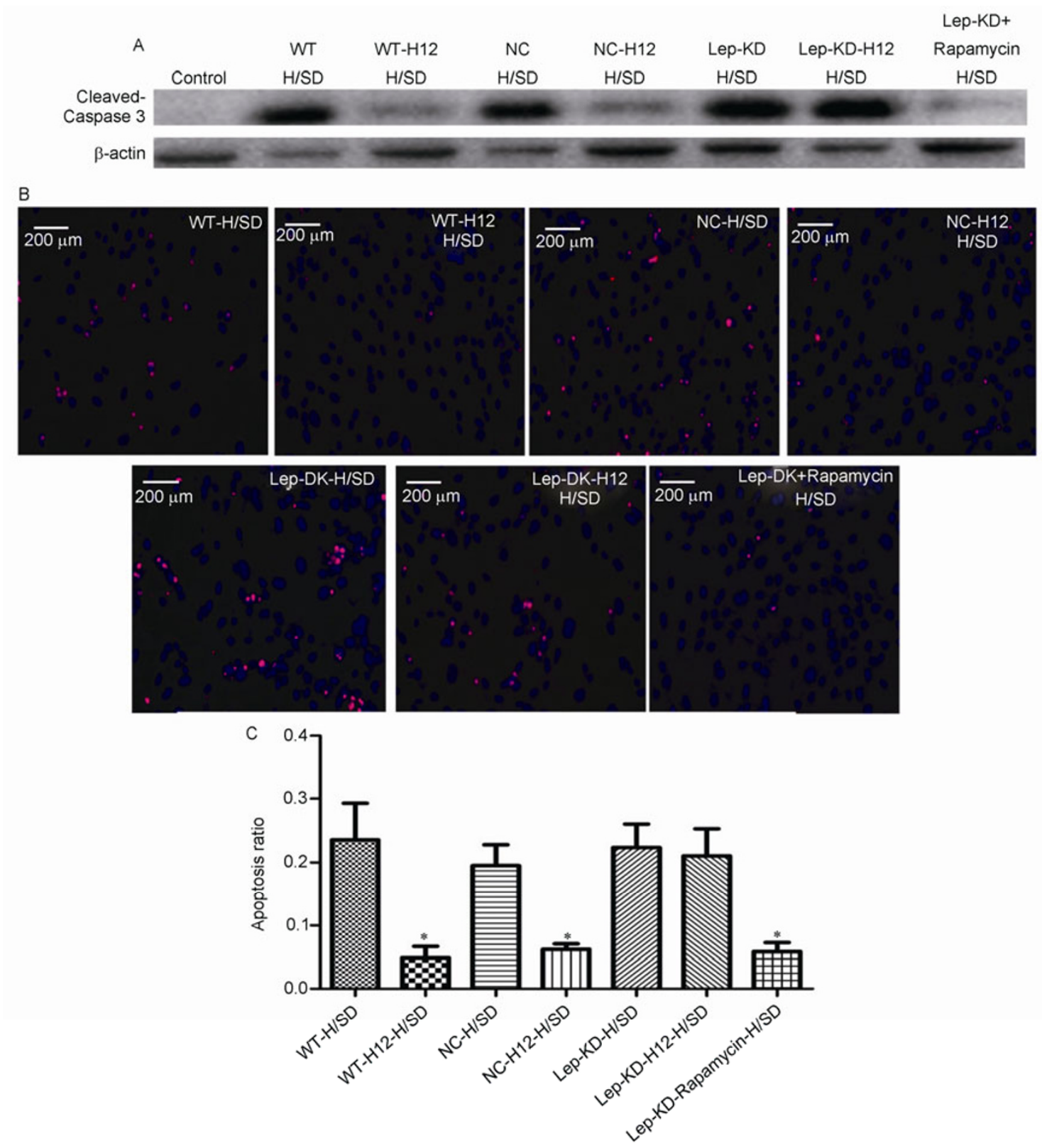

Figure 5 Leptin deficiency attenuates the protective effect of hypoxic-preconditioning. A, Western blotting analysis of levels of cleaved form of caspase 3 in BMSCs demonstrated that the protective effect of hypoxic-preconditioning was attenuated by leptin-knockdown. B, Terminal deoxynucleotidyl transferase dUTP nick end labelling (TUNEL) immunofluorescence analysis of BMSCs showed that hypoxic-preconditioning attenuated the apoptosis ratio of BMSCs under H/SD, while leptin-knockdown abrogated the protective effect. Fluorescent microscopic images show the TUNEL staining (red) and nuclear staining (blue). C, Quantification of apoptosis of BMSCs using the TUNEL stained images with Image Pro software. Figures are representative images of at least three different samples and each experiment was repeated at least thrice. Results are expressed as mean $\pm \mathrm{SD}$. *, $P<0.05$ vs. WT (wild type), NC (negative control), Lep-KD (leptin-knockdown) and Lep-KD-H12 (leptin-knockdown BMSCs treated with hypoxic-preconditioning for $12 \mathrm{~h}$ ).

cally induced by multiple stresses. The relationship between autophagy and death has been characterized in many contexts and has been discussed comprehensively; however, it has not been extensively studied in BMSCs. Hypoxia and serum deprivation are both energy-limiting stresses impairing the function of mitochondria and stimulating the production of radicals. According to numerous studies, adaptive autophagy is essential for cell survival by supplying energy and eliminating radicals and damaged organelles
[19-21]. The protective effect of autophagy has been previously reported in models of ischemic injury in the heart, brain, and under conditions of H/SD in cell culture [22-24]. Recently, Sanchez et al. [20] reported that during long period of serum deprivation, MSCs could utilize autophagy to provide needed energy and secret anti-apoptotic factors for survival. In our research we found that autophagic pathway was activated effectively by hypoxic-preconditioning as shown by increased autophagosomes, LC3-II, and decreased 


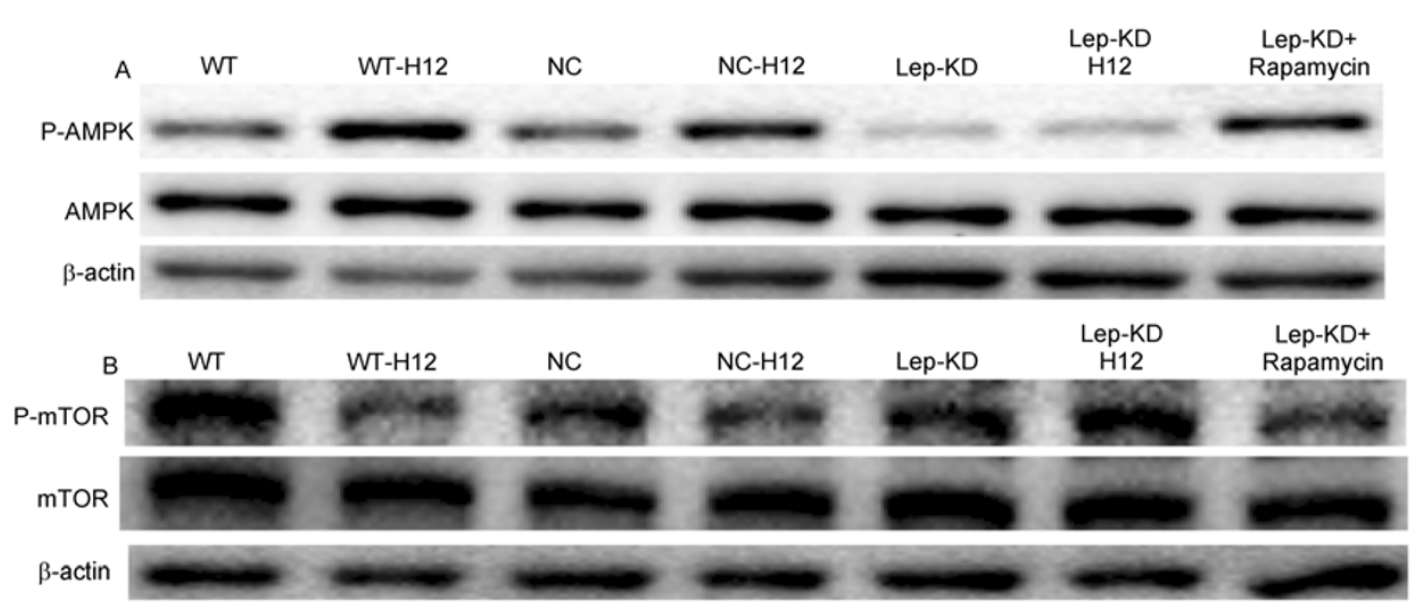

Figure 6 AMPK/mTOR pathway examined by Western blotting. A, Western blotting analysis showed that leptin-knockdown down-regulated the p-AMPK in BMSCs which was up-regulated by hypoxic-preconditioning. Meanwhile, rapamycin abrogated the negative effect of leptin deficiency. B, Leptin- knockdown up-regulated the p-mTOR in BMSCs which was down-regulated by hypoxic-preconditioning.

apoptosis ratio of BMSCs under H/SD. Moreover, suppressing autophagy by its inhibitor 3-MA resulted in increased apoptotic levels in H/SD-treated MSCs. In contrast, rapamycin, an autophagy inducer, could decrease the degree of apoptosis. These data suggested that HPC could promote autophagy in BMSCs as a pro-survival mechanism rather than a pro-death pathway in BMSCs under H/SD.

Interestingly, research data from our lab and others suggested that the expression of leptin was increased in MSCs after HPC. Leptin is a well-known molecule that promotes cell survival both in vivo and in vitro through various mechanisms $[25,26]$. In addition, recent research suggested that leptin induced autophagy in cultured human or mouse cell lines [13]. In this study conducted on BMSCs, we showed that leptin knockdown reduced HPC-induced autophagy by decreasing the LC3-II and autophagosomes. Autophagy promoter rapamycin abrogated these changes observed in the HPC-leptin-knockdown BMSCs group, suggesting that leptin does regulate HPC-induced autophagy. Furthermore, HPC-induced, autophagy mediated antiapoptosis effects on BMSCs under H/SD were significantly diminished by leptin-knockdown, while rapamycin reversed this leptin-knockdown effect. This confirmed our hypothesis that HPC induces autophagy through leptin and promotes BMSCs survival during H/SD.

It is noteworthy that in our study, the level of beclin1, a well-known autophagy-related protein, was not associated with the autophagic activity. However, as previous studies reported, the expression of beclin1 is augmented significantly when autophagy is induced $[22,27]$. This discrepancy may be due to the difference in cell types, stimuli, and duration of treatment applied to individual studies. Carloni et al. [28] have reported that applying hypoxia-ischemia stimulation for the same duration only induced beclin1 in neurons but not in other cells. Moreover, the treatment time associ- ated with autophaic markers' increase also differed widely from cell to cell. Thus, in our study, the short period of HPC may be the reason of lack of beclin 1 expression.

Up to now, many signaling pathways have been suggested in autophagy regulation. AMPK is thought to be an important positive regulator of autophagy via its indirect inhibition to mTOR [29]. Recent investigations suggested that leptin administration activates AMPK. Therefore, we monitored the expression and activation levels of AMPK/ mTOR pathway to further study the mechanism underlying HPC-induced autophagy. Interestingly, we found that HPC increased the AMPK activation and simultaneously reduced the mTOR activation significantly. Moreover, leptinknockdown not only counteracted the mTOR suppression and AMPK activation, but also eliminated the autophagy induced by HPC. In contrast, autophagy promoter rapamycin changed the signaling pathway of leptin-knock- down mediated by AMPK activation and mTOR, indicating that leptin and rapamycin may share the same signaling pathway in regulating autophagy in BMSCs, i.e., through mTOR inhibition. There may be many other mediators responsible for the autophagy. As other studies reported, leptin could also activate PI3K/Akt, STAT3, NF-kappaB, and ERK1/2 pathway to mediate the protection effects which are found to be able to signal autophagy as well [30-32]. Thus, further study is necessary to identify other signaling pathways used by leptin to regulate HPC-induced autophagy and protect BMSCs against apoptosis.

In conclusion, the results reported here strongly suggest that HPC induced autophagy in BMSCs protects BMSCs from apoptosis under H/SD. Leptin may be an important factor in regulating HPC-induced autophagy via AMPK/ mTOR pathway. These findings provide valuable information for better understanding of autophagy-regulated mechanisms that promote BMSCs survival. 
1 Hu X, Yu SP, Fraser JL, Lu Z, Ogle ME, Wang JA, Wei L. Transplantation of hypoxia-preconditioned mesenchymal stem cells improves infarcted heart function via enhanced survival of implanted cells and angiogenesis. J Thorac Cardiovasc Surg, 2008, 135: 799-808

2 Chang CP, Chio CC, Cheong CU, Chao CM, Cheng BC, Lin MT. Hypoxic preconditioning enhances the therapeutic potential of the secretome from cultured human mesenchymal stem cells in experimental traumatic brain injury. Clin Sci (Lond), 2013, 124: $165-176$

3 Choi AM, Ryter SW, Levine B. Autophagy in human health and disease. N Engl J Med, 2013, 368: 1845-1846

4 Li ZL, Lerman LO. Impaired myocardial autophagy linked to energy metabolism disorders. Autophagy, 2012, 8: 992-994

5 Zhang Q, Yang YJ, Wang H, Dong QT, Wang TJ, Qian HY, Xu H. Autophagy activation: a novel mechanism of atorvastatin to protect mesenchymal stem cells from hypoxia and serum deprivation via AMP-activated protein kinase/mammalian target of rapamycin pathway. Stem Cells Dev, 2012, 21: 1321-1332

$6 \mathrm{Hu}$ YL, DeLay M, Jahangiri A, Molinaro AM, Rose SD, Carbonell WS, Aghi MK. Hypoxia-induced autophagy promotes tumor cell survival and adaptation to antiangiogenic treatment in glioblastoma. Cancer Res, 2012, 72: 1773-1783

7 Wang B, Wood IS, Trayhurn P. Hypoxia induces leptin gene expression and secretion in human preadipocytes: differential effects of hypoxia on adipokine expression by preadipocytes. J Endocrinol, 2008, 198: 127-134

8 Grosfeld A, Andre J, Hauguel-De Mouzon S, Berra E, Pouyssegur J, Guerre-Millo M. Hypoxia-inducible factor 1 transactivates the human leptin gene promoter. J Biol Chem, 2002, 277: 42953-42957

9 Ahima RS, Flier JS. Leptin. Annu Rev Physiol, 2000, 62: 413-437

10 Myers MG, Cowley MA, Munzberg H. Mechanisms of leptin action and leptin resistance. Annu Rev Physiol, 2008, 70: 537-556

11 Minokoshi Y, Kim YB, Peroni OD, Fryer LG, Muller C, Carling D, Kahn BB. Leptin stimulates fatty-acid oxidation by activating AMP-activated protein kinase. Nature, 2002, 415: 339-343

12 Steinberg GR, Rush JW, Dyck DJ. AMPK expression and phosphorylation are increased in rodent muscle after chronic leptin treatment. Am J Physiol Endocrinol Metab, 2003, 284: E648-654

13 Malik SA, Marino G, BenYounes A, Shen S, Harper F, Maiuri MC, Kroemer G. Neuroendocrine regulation of autophagy by leptin. Cell Cycle, 2011, 10: 2917-2923

14 Mizushima N. Methods for monitoring autophagy. Int J Biochem Cell Biol, 2004, 36: 2491-2502

15 Knoepfler PS. Deconstructing stem cell tumorigenicity: a roadmap to safe regenerative medicine. Stem Cells, 2009, 27: 1050-1056

16 Ding DC, Shyu WC, Lin SZ. Mesenchymal stem cells. Cell Transplant, 2011, 20: 5-14

17 Lee J, Giordano S, Zhang J. Autophagy, mitochondria and oxidative stress: cross-talk and redox signalling. Biochem J, 2012, 441: 523-540

18 Mazure NM, Pouyssegur J. Hypoxia-induced autophagy: cell death or cell survival? Curr Opin Cell Biol, 2010, 22: 177-180

19 Zhang H, Bosch-Marce M, Shimoda LA, Tan YS, Baek JH, Wesley
JB, Gonzalez FJ, Semenza GL. Mitochondrial autophagy is an HIF-1-dependent adaptive metabolic response to hypoxia. J Biol Chem, 2008, 283: 10892-10903

20 Sanchez CG, Penfornis P, Oskowitz AZ, Boonjindasup AG, Cai DZ, Dhule SS, Rowan BG, Kelekar A, Krause DS, Pochampally RR. Activation of autophagy in mesenchymal stem cells provides tumor stromal support. Carcinogenesis, 2011, 32: 964-972

21 Hamacher-Brady A, Brady NR, Logue SE, Sayen MR, Jinno M, Kirshenbaum LA, Gottlieb RA, Gustafsson AB. Response to myocardial ischemia/reperfusion injury involves Bnip3 and autophagy. Cell Death Differ, 2007, 14: 146-157

22 Gurusamy N, Lekli I, Mukherjee S, Ray D, Ahsan MK, Gherghiceanu M, Popescu LM, Das DK. Cardioprotection by resveratrol: a novel mechanism via autophagy involving the mTORC2 pathway. Cardiovasc Res, 2010, 86: 103-112

23 Kanamori H, Takemura G, Goto K, Maruyama R, Ono K, Nagao K, Tsujimoto A, Ogino A, Takeyama T, Kawaguchi T, Watanabe T, Kawasaki M, Fujiwara T, Fujiwara H, Seishima M, Minatoguchi S. Autophagy limits acute myocardial infarction induced by permanent coronary artery occlusion. Am J Physiol Heart Circ Physiol, 2011, 300: H2261-2271

24 Loos B, Genade S, Ellis B, Lochner A, Engelbrecht AM. At the core of survival: autophagy delays the onset of both apoptotic and necrotic cell death in a model of ischemic cell injury. Exp Cell Res, 2011, 317: 1437-1453

25 Guo Z, Jiang H, Xu X, Duan W, Mattson MP. Leptin-mediated cell survival signaling in hippocampal neurons mediated by JAK STAT3 and mitochondrial stabilization. J Biol Chem, 2008, 283: 1754-1763

26 Magarinos MP, Sanchez-Margalet V, Kotler M, Calvo JC, Varone CL. Leptin promotes cell proliferation and survival of trophoblastic cells. Biol Reprod, 2007, 76: 203-210

27 Yu SW, Baek SH, Brennan RT, Bradley CJ, Park SK, Lee YS, Jun EJ, Lookingland KJ, Kim EK, Lee H, Goudreau JL, Kim SW. Autophagic death of adult hippocampal neural stem cells following insulin withdrawal. Stem Cells, 2008, 26: 2602-2610

28 Carloni S, Buonocore G, Balduini W. Protective role of autophagy in neonatal hypoxia-ischemia induced brain injury. Neurobiol Dis, 2008, 32: 329-339

29 Yang Z, Klionsky DJ. Mammalian autophagy: core molecular machinery and signaling regulation. Curr Opin Cell Biol, 2010, 22: 124-131

30 Wang D, Chen J, Chen H, Duan Z, Xu Q, Wei M, Wang L, Zhong M. Leptin regulates proliferation and apoptosis of colorectal carcinoma through PI3K/Akt/mTOR signalling pathway. J Biosci, 2012, 37: 91-101

31 Bates SH, Stearns WH, Dundon TA, Schubert M, Tso AW, Wang Y, Banks AS, Lavery HJ, Haq AK, Maratos-Flier E, Neel BG, Schwartz MW, Myers MG Jr. STAT3 signalling is required for leptin regulation of energy balance but not reproduction. Nature, 2003, 421: 856-859

32 Huang F, Xiong X, Wang $\mathrm{H}$, You S, Zeng H. Leptin-induced vascular smooth muscle cell proliferation via regulating cell cycle, activating ERK1/2 and NF-kappaB. Acta Biochim Biophys Sin (Shanghai), 2010, 42: 325-331

Open Access This article is distributed under the terms of the Creative Commons Attribution License which permits any use, distribution, and reproduction in any medium, provided the original author(s) and source are credited. 